\title{
On Systematic Model Reduction Techniques for Dynamic Optimization and Robust Control of Distributed Process Systems
}

\author{
Carlos Vilas, Míriam R. García, María R. Fernández, Eva Balsa-Canto, Julio R. Banga \\ and Antonio A. Alonso* \\ Process Engineering Group \\ IIM-CSIC, Eduardo Cabello 6, 36208 Vigo, Spain
}

\begin{abstract}
In this contribution we present an overview of some recent works carried out in our group to develop systematic and efficient methods for model reduction and its application to simulation, dynamic optimization and robust control of complex distributed process systems. The numerical projection methods we developed exploit the underlying finite element structure of the numerical PDE system to efficiently evaluate and to integrate the spatial differential terms, and thus to systematically project the original PDE set into a low dimensional subspace. This results into a reduced order description which is able to capture the relevant dynamics of the original system. Details on computational aspects of the methodology as well as applications in the context of dynamic optimization and robust control will be discussed on a number of representative case studies involving nonlinear diffusion-reaction and fluid dynamic systems.
\end{abstract}

Keywords: Distributed Process Systems, Reduced Order Models, Dynamic Optimization, Robust Control.

\section{Introduction}

Distributed Process Systems (DPS) emerge in process industry as a consequence of diffusion and convection mechanisms for mass, energy and momentum acting in the same time scale of process dynamics. Mathematically, this translates into a coupled and nonlinear set of partial differential equations (PDE's) describing the temporal and spatial distribution of property. The design of optimal operation policies for DPS requires the combination of efficient optimization and control tools with an appropriate system representation framework. In this way, the solution of a dynamic optimization problem, needs of an accurate process model to be solved at each objective function evaluation step. On the other hand, robust control methods rely on appropriate system descriptions able to capture its most relevant dynamic features (Alonso et al, 2002). The conventional approach to model and simulate DPS is based on spatial discretization schemes, which approximate the original PDEs by a large set of algebraic and ordinary

*antonio@iim.csic.es 
differential equations. However, the solution of the resulting system is computationally involved thus conditioning the efficiency of dynamic optimization algorithms. From a control design point of view, this approach presents also a number of disadvantages as some essential control-theoretic properties such as controllability or observability may be lost by the discretization scheme or the degree of refinement (Christofides, 2001). Alternative methodologies make use of the notion of dissipation to ensure the existence of a low dimensional subspace that captures most of the dynamic features of the original DPS (see Alonso et al, 2002; 2003). A finite dimensional dynamic representation, can then be obtained by projecting the original system of nonlinear partial differential equations on such a low dimensional subspace. However, a number of issues related with computational aspects of the spatial projection remain which prevent the extension of the methodology to nonlinear problems in complex spatial domains.

The purpose of this contribution is to provide an outline of an efficient numerical projection approach (Alonso et al, 2003; Balsa-Canto et al, 2002) that exploits the underlying finite element ( $\mathrm{fem}$ ) structure of the numerical PDE system to efficiently evaluate and to integrate the spatial differential terms, and thus to systematically produce consistent dynamic reduced order models (ROM). Details on the computational aspects of the methodology as well as applications in the context of dynamic optimization and robust control will be discussed on a number of representative case studies. These include: the efficient simulation of a 2D version of the Rayleigh-Bénard (natural convection) problem, the acceleration of the solution of an optimal control problem associated to a reaction-diffusion thermal process, and finally its application in the context of robust control to stabilize a class of dissipative systems known as Fitzhugh-Nagumo. The paper is organized as follows: A formal description of the methodology is presented in Section 2, while Section 3 offers, on a number of subsections, a detailed discussion of the three types of case studies.

\section{Theoretical aspects of model reduction}

The class of systems considered in this work (distributed process systems) can be represented by a general equation of the form:

$x_{t}+\nabla\left(v_{k} x\right)=\kappa \Delta x+f(t, \xi, x, u)$

where $x$ is a the vector of the state variables (the field), $\xi$ the spatial coordinates, $\nabla$ and $\Delta$ are the well-known gradient and Laplacian operators, respectively, and $u$ the control. The main consequence of dissipation conditions is that the field $\mathrm{x}$ can be represented as an infinite series expansion of the form:

$\mathrm{x}(\mathrm{t}, \xi)=\sum_{\mathrm{j}=1}^{\infty} \mathrm{c}_{\mathrm{j}}(\mathrm{t}) \varphi_{\mathrm{j}}(\xi)$,

where each element of the set $\left\{\varphi_{\mathrm{j}}\right\}_{\mathrm{j}=1}^{\infty}$ of equation (2) is calculated as the solution of:

$\varphi_{\mathrm{j}}(\xi)=\frac{1}{\lambda_{\mathrm{j}}} \int_{\Omega} \mathrm{R}\left(\xi, \xi^{\prime}\right) \varphi_{\mathrm{j}}\left(\xi^{\prime}\right) \mathrm{d} \xi^{\prime}$, 
with $\lambda_{\mathrm{j}}$ being a parameter associated with each basis $\varphi_{\mathrm{j}}$. Under the orthonormality condition, and depending on the nature of the kernel R, two sets can be considered: spectral decomposition, if $\mathrm{R}$ is the Green function associated with the spatial operators $\Delta$ and $\nabla$ (Courant and Hilbert, 1937) or proper orthogonal decomposition, if $\mathrm{R}$ corresponds with the two point correlation matrix (Balsa-Canto et al., 2002). The ordered structure of the eigenspectrum $\left\{\lambda_{\mathrm{j}}\right\}_{\mathrm{j}=1}^{\infty}$ is then used to select the finite low dimensional basis $\Phi=\left[\varphi_{1} \vdots \varphi_{\mathrm{N}}\right]$ which will capture the most relevant features of the original system. By projecting system (1) on each function $\varphi_{\mathrm{j}}$ of the basis $\Phi$, the following set of ODEs is obtained:

$\mathrm{c}_{\mathrm{t}}=\mathrm{Ac}+\mathrm{F}$

$\widetilde{\mathrm{x}}=\Phi \mathrm{c}$

where $\mathrm{A}$ and $\mathrm{F}$ are the projection of spatial operators and non-linear terms respectively. The Galërkin projection of the general structure (1) onto the finite elements basis functions $\left\{\phi_{\mathrm{j}}\right\}_{\mathrm{j}=1}^{\mathrm{N}}$ can be represented as:

$\mathrm{DAc}_{\mathrm{t}}(\mathrm{t})+(\mathrm{C}+\mathrm{Q}+\mathrm{BE}) \mathrm{c}(\mathrm{t})=\mathrm{F}+\mathrm{G}$

where $\mathrm{DA}=<\phi_{\mathrm{i}}, \phi_{\mathrm{j}}>_{\Omega}, \mathrm{C}=<\kappa \nabla \phi_{\mathrm{i}}, \nabla \phi_{\mathrm{j}}>_{\Omega}, \mathrm{BE}=<\mathrm{v}_{\mathrm{k}} \nabla \phi_{\mathrm{i}}, \phi_{\mathrm{j}}>_{\Omega}, \mathrm{F}=<\mathrm{f}, \phi_{\mathrm{i}}>_{\Omega}$, with $<\cdot, \cdot>_{\Omega}$ representing the inner product of two vector valued functions defined on the domain $\Omega$, and $\mathrm{Q}$ and $\mathrm{G}$ are related to the boundary conditions. The f.e.m. structure allows spatial derivatives and integrals to be approximated by algebraic operations which makes the task of projection straightforward. Such operations are carried out according to the following criterion:

Let $\mathrm{P}$ be any of the matrices DA, BE or C, calculated with parameters $\kappa$ and $\mathrm{v}_{\mathrm{k}}$ equal to unity, and $\Pi$ their corresponding operator (gradient for BE, Laplacian for $\mathrm{C}$ and the unity for DA). Integration of any two functions $f$ and $g$ on the spatial domain $\Omega$ and spatial differentiation of a function $\mathrm{f}$ are given, respectively, by:

$\int_{\Omega} \mathrm{f} \Pi g \mathrm{~d} \Omega=\mathrm{f}^{\mathrm{T}} \mathrm{Pg} ; \quad \Pi \mathrm{f}=\mathrm{DA}^{-1} \mathrm{Pf}$

\section{Applications}

\subsection{Simulation of 2D version of Rayleigh-Bénard}

The dimensionless form of the Rayleigh-Bénard system is given by the following set of equations with appropriate boundary conditions (Hung and Hien, 2001):

$\gamma \frac{\partial \overrightarrow{\mathrm{u}}}{\partial \mathrm{t}}+\gamma \overrightarrow{\mathrm{u}} \nabla \overrightarrow{\mathrm{u}}=-\nabla \mathrm{P}+\gamma \overrightarrow{\mathrm{j}} \mathrm{T}+\Delta \overrightarrow{\mathrm{u}}$

$\gamma \frac{\partial \mathrm{T}}{\partial \mathrm{t}}+\gamma \overrightarrow{\mathrm{u}} \nabla \mathrm{T}=\Delta \mathrm{T}$

$\nabla \overrightarrow{\mathrm{u}}=0$ 
where $\gamma=\sqrt{\operatorname{Ra} / \operatorname{Pr}}, \overrightarrow{\mathrm{u}}$ is the fluid velocity, $\mathrm{T}$ the temperature and $\mathrm{P}$ the pressure. A low dimensional system of ODEs of the form (4) is obtained by projecting system (7) on each function of the set $\Phi$, computed through the POD method. The dimension of the resulting ODE set which is able to produce an accurate description of the dynamics is two orders of magnitude smaller. The level of accuracy can be seen in Figure 1, where the temperature distribution computed by direct numerical simulation -Figure 1(a)- and that obtained with the ROM -Figure 1(b)- are represented.

(a)

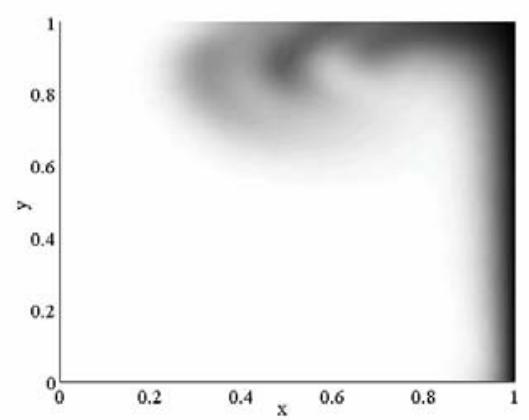

(b)

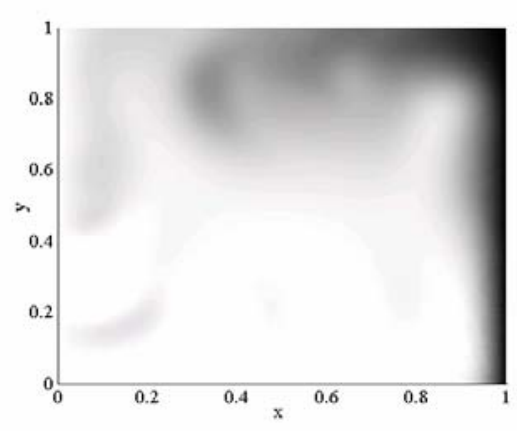

Figure 1: Temperature distribution calculated with (a) finite elements and (b) ROM.

\subsection{Optimal control of nonlinear distributed systems}

The open loop optimal control (OC) problem is formulated as the calculation of the optimal operating conditions to achieve a certain goal subject to the system dynamics and possibly other constraints. In this work the OC of the thermal sterilization of canned foods was considered (Balsa-Canto et al, 2002). The objective was to calculate the optimal time dependent processing temperature in order to maximize nutrient retention while guarantying food safety as formulated in Banga et al. (1991). The control vector parameterization approach (CVP) (Vassiliadis, 1993) combined with a global optimization method (ICRS/DS, (Banga et al.,1998)) were used for the solution of the OC problem. It must be remarked that most of the computational effort for the solution of the OC problem is devoted to the process model simulation. Therefore it is of the highest interest to obtain a reduced order description to decrease the computational cost and thus enable real time applications. The performance of the reduced and a nominal (full) model obtained by using the numerical method of lines were compared so as to test the ability of the ROM to capture the relevant dynamic features of the system. As illustrated in Figure 2(a) the results are indistinguishable.

Figure 2(b) presents the convergence curves of both optimization processes showing the superiority in terms of efficiency of the reduced model. By using the ROM, a retention value very close to the optimum was reached in only $0,27 \mathrm{~s}$ while the computational effort to obtain the final (global) solution was up to 15 times smaller. These results demonstrate that ROMs can be used not only for the efficient solution of $\mathrm{OCP}$, but also in applications such as real time optimization or model predictive control. 
(a)

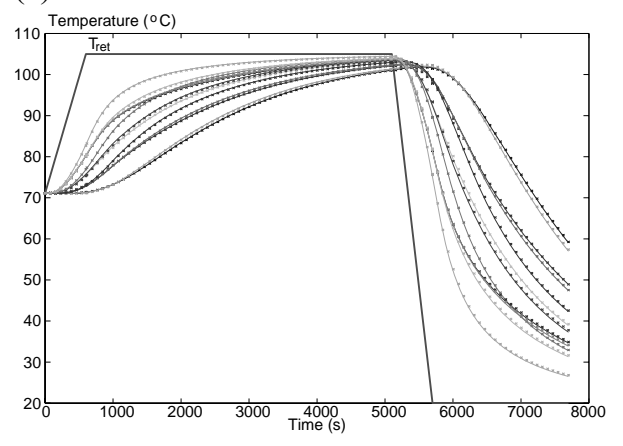

(b)

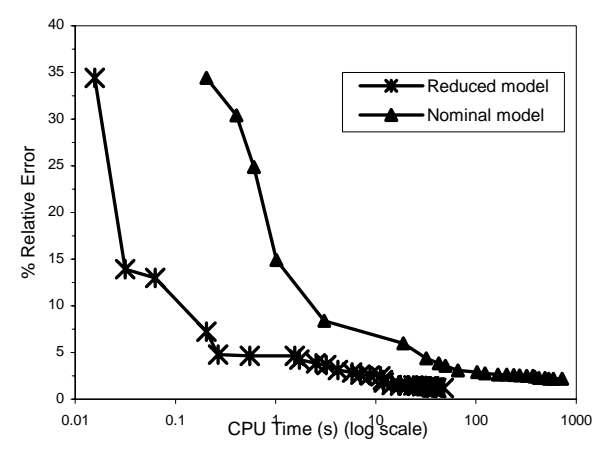

Figure 2: (a) Comparison of the reduced (dots) and the nominal (lines) models; (b) Comparison of the curves of convergence for the OC.

\subsection{Robust control of the Fitzhugh-Nagumo problem}

A two species version of the Fitzhugh-Nagumo model (see for instance, Murray, 1993), can be described by the following set of PDEs:

$$
\begin{aligned}
& \mathrm{v}_{\mathrm{t}}=\kappa \Delta \mathrm{v}+\mathrm{F}(\mathrm{v})-\mathrm{w}=\kappa \Delta \mathrm{v}+\mathrm{v}-\mathrm{v}^{3}-\mathrm{w}+\pi \\
& \mathrm{w}_{\mathrm{t}}=\delta \kappa \Delta \mathrm{w}+-\varepsilon \mathrm{G}(\mathrm{w})+\varepsilon \mathrm{v}=\delta \kappa \Delta \mathrm{w}+\varepsilon\left(\mathrm{v}-\mathrm{p}_{1} \mathrm{w}-\mathrm{p}_{0}\right)
\end{aligned}
$$

where $\mathrm{v}$ and $\mathrm{w}$ are activator and inhibitor species. $\delta$ and $\varepsilon$ are, respectively, the ratio of diffusion coefficients and reaction rates for the two species, and $\pi$ the control. As shown in Alonso et al (2003), this system is dissipative with respect a stationary state of reference $\mathrm{v}^{*}=\mathrm{w}^{*}=0$. This property allows the fields $\left(\mathrm{v}=\mathrm{v}-\mathrm{v}^{*}\right.$ and $\left.\mathrm{w}=\mathrm{w}-\mathrm{w}^{*}\right)$ to be partitioned in sub-fields as:

$\nabla=\nabla_{1}+\nabla_{2}=\sum_{j=1}^{\infty} c_{v j}(t) \varphi_{j}(\xi) ; \quad w=w_{1}+w_{2}=\sum_{j=1}^{\infty} c_{w j}(t) \varphi_{j}(\xi)$

with $\varphi_{\mathrm{j}}$ satisfying equation (3) and $\mathrm{R}$ being the Green function associated to the Laplacian operator. In equation (9), $\left(\mathrm{\nabla}_{2}, \mathrm{w}_{2}\right)$ are associated to a finite dimensional set $\mathrm{S}_{2}=\left\{\varphi_{\mathrm{j}}\right\}_{\mathrm{j}}$ with their corresponding eigenvalues $\Lambda_{2}=\left\{\lambda_{\mathrm{j}}\right\}_{\mathrm{j}}$ and $\left(\nabla_{1}, \mathrm{~W}_{1}\right)$ are associated to its infinite dimensional complement $\left(\mathrm{S}_{1}, \Lambda_{1}\right)$. A robust control scheme which takes advantage of the underlying reduced dynamics has been implemented so to stabilize the subsystem $\mathrm{S}_{1}$, while preserving the inherent oscillatory dynamics of the uncontrolled modes. The resulting (ultimately bounded) control law is of the form:

$\pi=\pi_{1}=\left\{\begin{array}{ccc}\mathrm{w}_{1}-\omega \nabla_{1}-\frac{\eta}{\left\|\nabla_{1}\right\|_{\Omega}} & \text { si } & \eta \nabla_{1} \|_{\Omega} \geq \varepsilon \\ \mathrm{w}_{1}-\omega \nabla_{1}-\frac{\eta^{2}}{\varepsilon} & \text { si } & \eta\left\|\nabla_{1}\right\|_{\Omega}<\varepsilon\end{array}\right.$

The performance of this controller is illustrated in Figure 3. The open loop unstable modes presented in Figure 3(a) become ultimately bounded -Figure 3(b)- under the robust control law (10). 
(a)

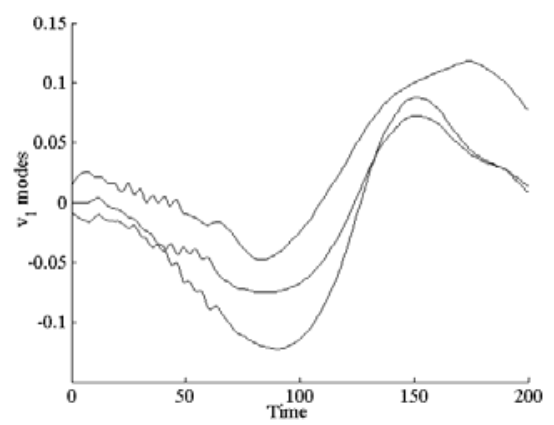

(b)

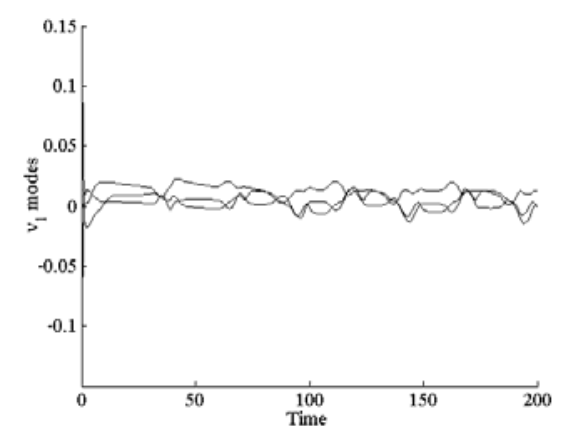

Figure 3: Representation of $\mathrm{v}_{1}$ modes: (a) open loop $\left(\pi_{1}=0\right)$ (b) with control law of the form (10).

\section{Conclusions}

In this work, we presented a systematic and efficient methodology which takes advantage of the underlying finite element structure to develop reduced order descriptions for PDE dissipative systems which retain the relevant (slow) nonlinear dynamics. The numerical aspects of this approach have been discussed and illustrated on a number of relevant applications involving complex phenomena such as nonlinear diffusion-reaction and fluid dynamics.

\section{References}

Alonso, A.A. J.R. Banga and B.E. Ydstie, 2002, From irreversible thermodynamics to robust control theory for distributed process systems. J. Proc. Control., 12, 507-515.

Alonso, A.A., C.V. Fernández and J.R. Banga, 2004, Dissipative systems: from physics to robust nonlinear control. Int. J. of Robust and Nonlinear Cont., 14, 157-179.

Balsa-Canto, E., A.A. Alonso and J.R. Banga, 2002, A novel, efficient and reliable method for thermal process design and optimization. Part I: theory. J. Food Eng., 52, $3,227-234$.

Banga, J. R., R. Irizarry, and W. D. Seider, 1998. Stochastic optimization for optimal and model-predictive control. Comp. Chem. Eng., 22(4-5):603-612.

Christofides, P.D., 2001, Nonlinear and Robust Control of PDE Systems. Birhauser, Boston.

Courant, R. and D. Hilbert, 1937. Methods of Mathematical Physics. Wiley, New York.

Hung, V.L. and T.T. Hien, 2001, Modelling and control of physical processes using proper orthogonal decomposition. Math. and Comp. Modelling, 33, 223-236.

Murray, J.D., 1993, Mathematical Biology. Second edition. Vol 19. Springer.

Vassiliadis, V. S. Computational Solution of Dynamic Optimization Problems with General Differential-Algebraic Constraints. PhD thesis, Imperial College, University of London, U.K.,July 1993. 